\title{
Research on Collaborative Innovation Strategy of Smart Supply Chain in the Big Data Era
}

\author{
He Wang ${ }^{1, a}$, Xiang Huang ${ }^{2, b}$ \\ ${ }^{1}$ IFLYTEK CO.LTD., Hefei, China \\ ${ }^{2}$ Hefei Institute of Physical Science, Chinese Academy of Sciences, Hefei, China
}

\begin{abstract}
Artificial intelligence, big data, Cloud computing and IOT, technology accelerates integration and innovation, and data dividends have continued to emerge. At the same time, China's "Internet Action Plan", "Made in China 2025", "Digital China" and other national strategies have been implemented in depth, China's social and economic development has entered the era of big data. As the basic industry of the national economy. The logistics industry will also accelerate changes, and it has become a development trend for companies to use new technologies to realize smart supply chain collaborative innovation. The paper analyzes the development opportunities of smart supply chain in the Big Data Era, summarizes the problems encountered in the application of big data in the smart supply chain at this stage, and finally puts forward the collaborative innovation strategy for smart supply chain in the Big Data Era, Providing reference for collaborative innovation development of enterprise supply chain.
\end{abstract}

\section{INTRODUCTION}

The continuous progress of science and technology and the continuous deepening of social information, Smart applications based on big data and artificial intelligence will realize the intelligence of all things. In this process, data has become the fifth new production factor after land, labor, capital and technology. The resulting digital economy has become a driving economy. New momentum for growth and social progress. In the context of big data, the value of data is valued, explored and utilized, and it has become an effective means for people to make scientific decisions. As the company's "third profit source" logistics will deeply integrate big data technology and use big data as its strategic resource. Enterprises can use big data analysis to deeply explore the information value behind the data, help improve the level of operation and management, and improve the efficiency of logistics supply chain operations. Enterprises should innovate supply chain management models, create a customer-centric, big data-driven smart supply chain, and improve logistics service level and user experience, providing support for scientific management and decision-making of enterprises.

\section{DEVELOPMENT OPPORTUNITIES FOR SMART SUPPLY CHAINS IN THE BIG DATA ERA}

\section{A. Wide application of new technology}

Through the Hype Cycle for Supply Chain Strategy data 2020, source from Gartner. In the next few years, emerging new technology Such as Data technology, Artificial intelligence, Block chain and the IOT have brought new changes to the development of the logistics industry, and promoted the development of the industry toward intelligence and interconnection. The era of smart supply chain is coming. Information system construction, data collaboration, and the popularization of handheld terminals will significantly increase the level of logistics digitization, breaking industry information asymmetry and information islands, and logistics data showing an exponential growth trend, forming a fully-covered and widely-connected logistics Internet, "Internet of Everything" Promote the development of smart supply chain. At the same time, with the in-depth advancement of the Industrial Internet, logistics robots will replace humans in many aspects, and technology empowerment will transform traditional logistics genes, and the "intelligent revolution" will change the pattern of smart logistics. The wide application of various new technologies can extract valuable information from massive logistics data and realize the collaborative and innovative development of the smart supply chain. 


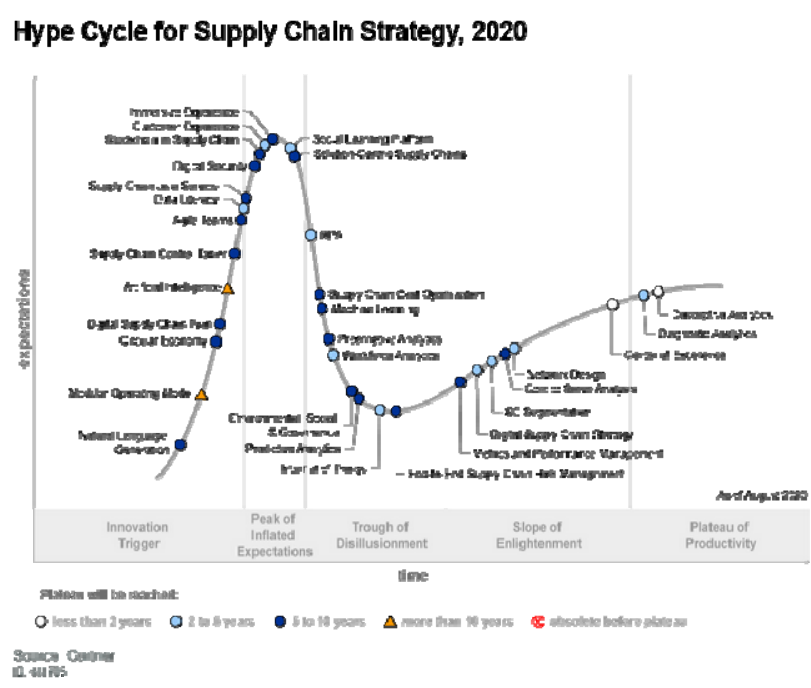

Figure 1. Supply Chain Program Technology Maturity Curve, 2020(Source:Gartner)

\section{B. Information interconnection}

Based on its own information advantages, big data will realize the unprecedented interactive capabilities of the smart supply chain, which not only includes the interactive connection of the information of all node companies in the supply chain, but also realizes the comprehensive multi-channel connection of all goods in the smart supply chain. Big data transforms the static goods circulation on the smart supply chain into dynamic data information, and realizes the effective interaction between the node enterprises on the supply chain through the dynamic data of goods. The supply chain, manufacturing enterprises, and customers form a close connection to realize the close integration of consumers and manufacturers. Through the mining and processing of big data, it is possible to support front-end R\&D and production based on consumer data, effectively reducing The reaction time of the chain and save supply chain costs, realize the interactive connection of suppliers, enterprises, third-party logistics, customers, etc., and realize a fully connected smart supply chain network.

\section{Intelligent processing of data}

The smart supply chain is empowered by big data technology to create a smart supply chain brain system. Through data mining and artificial intelligence technology, it can realize self-intelligent learning. It can be based on the actual situation at the time without manual intervention. Make corresponding decisions. The smart supply chain uses data analysis to predict what will happen in the future. It collects information such as online and offline receipts, sales data and uses mathematical modeling and mathematical algorithms to analyze and forecast consumer needs, classify consumer groups, and achieve accurate consumer demand forecasts for each consumer group to achieve precise marketing. The value of data in the era of big data will be fully tapped with the help of artificial intelligence technology.

\section{Problem Research on the DEVELOPMENT OF SMART SUPPLY CHAINS IN THE BIG DATA ERA}

\section{A. The problem of Enterprise's emphasis on big data}

In the era of big data, the market competition faced by enterprises is becoming more and more fierce, and the continuous improvement of the level of competition makes enterprises have more and more demand for data information. When enterprises need to obtain high-quality decisions, The traditional data used for decision-making is far from meeting this demand. In the era of big data, seemingly irrelevant information may have a high degree of value, thus affecting the decision-making of enterprises. Therefore, in the era of big data, companies need to have a wide range of information for decision-making. This requires companies to speed up the collection, sorting and analysis of data, and to fully mine and analyze the vast information and data to make them their own Data assets. In the era of big data, enterprises should attach importance to the value of data, and make value judgments based on the results of big data analysis in decision-making, seize opportunities and challenges, conduct business management with big data thinking, and enhance the core competition of the enterprise Advantage.

\section{B. The problem of Enterprise Big Data Scientific planning}

With the in-depth application of big data in the field of logistics supply chain, the value of big data in supply chain management has been valued by more and more enterprises. But to solve the problem of data application, how to make the data generated in the conversion process of each link of the supply chain have commercial value, is the foundation of the revolutionary productivity of data deployment. The application of big data in the supply chain is no longer simply a matter of visualizing transaction status and supporting decision-making inventory levels, which traditional ERP systems cannot afford. Therefore, enterprises must re-do the top-level design of logistics data application and establish a powerful and comprehensive big data application analysis model to deal with the challenge of how to play the value of complex and massive data.

\section{The problem of promotion intelligent service level}

In the big data era, a customer-centric supply chain model has been developed. Companies are facing more fierce competition. Delivery times are constantly shortening, product quality is constantly improving, and operational efficiency is continuously improving. Companies are expected to deliver personalized delivery in a short period of time, and can achieve profitability. The delivery time of enterprises is constantly being compressed, the variables in product design are 
constantly increasing, and customer needs may change at any time. These market conditions have brought huge operational pressures to companies. To deal with these challenges, the only way is to build an end-to-end digital supply chain. By establishing a new ecosystem across different regions and industries, the system network can generate data and integrate these data with internal enterprise data and product data across organizational structures, and intelligently analyze the data with the help of artificial intelligence technology to truly realize the intelligent management of the smart supply chain.

\section{Supply chain data security issues}

With the rapid development of information technology, our country is paying more and more attention to information security, and information security technology is becoming more and more mature. However, supply chain data security is related to the entire process system. The actual operation process still needs to be vigilant to ensure that no data security accidents occur. Supply chain data security risks are determined by the complexity of the logistics supply chain links. All links in the enterprise supply chain based on big data technology are interlocking, they depend on each other and affect each other. Problems occur in any of these links. It may affect other links and affect the normal operation of the entire supply chain. Supply chain data security risk is a potential threat, which not only causes damage to the processes of each link in the supply chain, but also causes systematic damage and loss to the entire supply chain. The more enterprises rely on supply chain data, the more attention must be paid to data security to ensure that the data in the enterprise smart supply chain is absolutely safe and effective.

\section{Collaborative INNOVATION STRATEgy OF SMART SUPPLY CHAIN IN THE BIG DATA ERA}

\section{A. Create a smart supply chain collaborative innovation platform}

Now that we have reached the era of digital transformation of the supply chain, traditional ERP can no longer meet the needs of current social development. It must extend to both ends of the entire enterprise and extend to both ends of the supply chain on the basis of enterprise ERP. On the one hand, according to a unified data standard and architecture system, enterprises integrate upstream and downstream ecological partners, open up the entire supply chain, share data, share risks, continue development, and collaborate for a win-win situation, and jointly create a shared platform for smart supply chain operations. On the other hand, through the digital platform to integrate the upstream and downstream of the supply chain, the upstream and downstream enterprises of the entire supply chain can be connected, and the integration of information flow and business process and information sharing can be realized, so that each process can understand the supply chain operation status. At the same time enterprise realize the connection between operational production data and market sales data. Using artificial intelligence technology to conduct big data analysis, enterprise realize market forecasts, and create a smart supply chain innovation system centered on market customers, as shown in Figure 2.

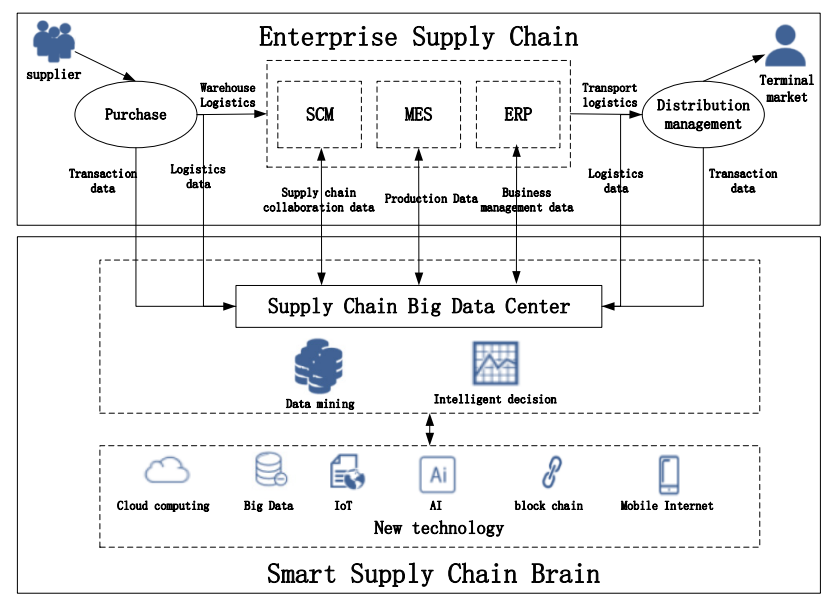

Figure 2. Architecture diagram of smart supply chain data collaboration platform

\section{B. Cultivate compound smart supply chain talents}

In the era of big data, the logistics industry is moving towards intelligence and digitization, which places higher requirements on talents in the logistics field. Enterprises should pay attention to cultivating compound talents who understand both logistics business and new logistics technologies In order to meet the needs of collaborative development of enterprise smart supply chain. On the one hand, it is necessary to look for high-end logistics Information talents according to the development requirements of the enterprise. They can lead the enterprise to carry out the overall planning of logistics informatization and do a good job in the top-level design of informatization, in order to realize the scientific and orderly development of enterprise logistics informatization. On the other hand, we must also actively cooperate with universities to integrate production, education and research, and promote the cultivation of compound talents based on the development needs of enterprises. At the same time, it is necessary to search for and discover potential talents from enterprise internal, we should formulate relevant training plans, strengthen employee's logistics management and intelligent technical ability, gradually improve their professional skills, in order to make them grow quickly into the backbone of the company.

\section{Establish smart supply chain data security management system}

In the big data era, enterprises must pay more attention to data security management, establish a smart supply chain data security management system, and enhance the 
awareness of data security protection of logistics operators. First of all, enterprises must strengthen the data information management of the entire supply chain, and at the same time ensure that the information of the supply chain ecological cooperation enterprises will not be stolen, will not be lost, and ensure data security. Secondly, according to the characteristics of shared data in the smart supply chain, big data technology is effectively used to promote effective collaboration in the collection, sorting, and transformation of information in all links of the supply chain, so as to provide a reference for the efficient operation of the enterprise. To ensure that data brings the advantages of collaborative innovation in the supply chain to the enterprise, it also meets the data security requirements of smart supply chain.

\section{CONCLUSION}

In the context of big data, digital technology is subverting the traditional supply chain development model. The smart supply chain has gradually developed from the initial concept to mature applications, from the manufacturing industry to the entire industry. Smart supply chain is the future development trend and has become the industry consensus. This has brought a new round of development opportunities to companies all over the world. If an enterprise wants to seize the opportunity for development, it must follow the trend and use big data technology to upgrade the traditional supply chain model and transform to a smart supply chain in order to stand out in the fierce market competition.

\section{REFERENCES}

1. Di Xiao, Xiansheng Kuang, Kebing Chen E-commerce supply chain decisions under platform digital empowerment-induced demand[J] Computers \&amp; Industrial Engineering, 2020, 150.

2. Olatunde A. Durowoju, Hing Kai Chan, Xiaojun Wang et al. Supply chain redesign implications to information disruption impact[J] International Journal of Production Economics, 2020.

3. Wang Bill, Luo Wen, Zhang Abraham et al. Blockchain-enabled circular supply chain management: A system architecture for fast fashion[J] Computers in Industry, 2020, 123.

4. Digitalisation and work: challenges from the platform-economy $[\mathrm{J}]$ Contemporary Social Science, 2020, 15(4).

5. Swapnil Lahane, Ravi Kant, Ravi Shankar Circular supply chain management: A state-of-art review and future opportunities $[\mathrm{J}]$ Journal of Cleaner Production, 2020, 258

6. Terrada Loubna, Khaïli Mohamed El, Ouajji Hassan MultiAgents System Implementation for Supply Chain Management Making-Decision[J] Procedia Computer Science, 2020, 177.

7. Swapnil Lahane, Ravi Kant, Ravi Shankar Circular supply chain management: A state-of-art review and future opportunities[J] Journal of Cleaner Production, 2020, 258. 